\title{
Effects of Pt content on the microstructure and magnetic properties of CoTbPt thin films
}

\author{
P. C. Kuo a) and C. T. Lie \\ Institute of Materials Science and Engineering, National Taiwan University, Taipei 106, Taiwan \\ S. C. Chen \\ Department of Mechanical Engineering, De Lin Institute of Technology, Taipei 236, Taiwan \\ C. Y. Chou, T. H. Wu, and L. Y. Chang \\ Institute of Materials Science and Engineering, National Taiwan University, Taipei 106, Taiwan
}

(Presented on 14 November 2002)

\begin{abstract}
This work presents a single-layer $\mathrm{CoTbPt}$ film for use as a hybrid-recording medium. $\mathrm{Co}_{77.5-x} \mathrm{~Tb}_{22.5} \mathrm{Pt}_{x}$ films with $x=0-14$ at. \% are fabricated on glass and naturally oxidized silicon wafer substrates by de magnetron sputtering. The magnetic film is sandwiched between $\operatorname{SiN}_{x}$ protective layers to prevent oxidization. The effects of Pt content on the magnetic properties and microstructure of the film are investigated. X-ray diffraction and transmission electron microscope diffraction patterns revealed that all the films are amorphous. It shows that substituting a small amount of $\mathrm{Co}$ for the nonmagnetic element $\mathrm{Pt}$ will increase the saturation magnetization, the perpendicular remanence, and the perpendicular coercivity of the $\mathrm{Co}_{77.5} \mathrm{~Tb}_{22.5}$ film. It is found that the saturation magnetization of the $\mathrm{Co}_{75.3} \mathrm{~Tb}_{22.5} \mathrm{Pt}_{2.2}$ film is about $245 \mathrm{emu} / \mathrm{cm}^{3}$, the perpendicular squareness is about 0.8 , perpendicular coercivity is about 2100 Oe at room temperature and the coercivity decreases rapidly with increasing temperature. The coercivity is decreased to about $50 \mathrm{Oe}$ when temperature is increased to $200{ }^{\circ} \mathrm{C}$. (C) 2003 American Institute of Physics.
\end{abstract}

[DOI: $10.1063 / 1.1555796]$

\section{INTRODUCTION}

The hybrid-recording method, which combines thermally assisted writing and magnetic flux detection, has been suggested to increase the recording density of magnetic disks. ${ }^{1-3}$ In this method, the small perpendicular domains are formed by thermomagnetic writing and detected by a highresolution giant magnetoresistive (GMR) head or a tunneling magnetoresistive (TMR) head.

The recording media for hybrid recording must satisfy three requirements: (1) they must provide satisfactory magneto-optical writing performance; (2) they must have a large saturation magnetization, $M_{s}$, that generates sufficient magnetic flux for the GMR head or the TMR head readout, and (3) they must have a large perpendicular coercivity, $H_{c}$, to resist self-demagnetization. The traditional magnetooptical (MO) recording medium has been considered for use as a hybrid recording medium because of its writing principle is the same as that of hybrid recording. However, the $M_{s}$ value of the MO medium at room temperature is too small to generate sufficient magnetic flux for GMR or TMR head readout. The bilayer structure, including a readout layer and a recording layer, is proposed to solve this problem. ${ }^{4,5}$ The recording layer has good thermomagnetic writing characteristics and a high $H_{c}$ at room temperature. The readout layer has a large $M_{s}$ at room temperature, and thus generates sufficient flux density for GMR or TMR head sensing.

This study aims to find a single-layer recording medium which is suitable for a hybrid-recording medium application.

\footnotetext{
${ }^{a)}$ Electronic mail: pckuo@ccms.ntu.edu.tw
}

Previously, we showed that the magnetic properties of amorphous CoTb film are sensitive to the composition of the film and the process parameters. ${ }^{6}$ The perpendicular coercivities of some amorphous CoTb films are very large, but their $M_{s}$ values are small at room temperature. This work incorporates $\mathrm{Pt}$ in the CoTb alloy film to increase its $M_{s}$ value. The effects of Pt content on the structure and magnetic properties of the CoTbPt film are also investigated.

\section{EXPERIMENT}

$\mathrm{Co}_{77.5-x} \mathrm{~Tb}_{22.5} \mathrm{Pt}_{x}$ films with $x=0-14$ at. $\%$, were deposited on glass and on naturally oxidized $\mathrm{Si}(100)$ substrates by dc magnetron sputtering at room temperature. The target was a Co disk overlaid with $\mathrm{Tb}$ and Pt pieces to obtain a desired film composition. The base pressure was 5 $\times 10^{-7}$ Torr and the sputtering pressure of Ar gas was maintained at 4 mTorr. The magnetic film was sandwiched between $\mathrm{SiN}_{x}$ protective layers to prevent oxidization. The $\mathrm{SiN}_{x}$ protective layers were produced by rf magnetron sputtering of an $\mathrm{Si}_{3} \mathrm{~N}_{4}$ target. The thicknesses of the $\mathrm{Co}_{77.5-x} \mathrm{~Tb}_{22.5} \mathrm{Pt}_{x}$ layer and the $\mathrm{SiN}_{x}$ layer were $75 \mathrm{~nm}$ and $30 \mathrm{~nm}$, respectively.

The microstructures of the films were examined by x-ray diffraction (XRD) with $\mathrm{Cu} K \alpha$ radiation, and by transmission electron microscopy (TEM). Depth profiles of the elements in the films were analyzed by Auger electron spectroscopy (AES). Compositions of the films were determined by $\mathbf{x}$-ray energy dispersive spectroscopy. The magnetic properties of the films were measured using a vibrating sample magnetometer. 


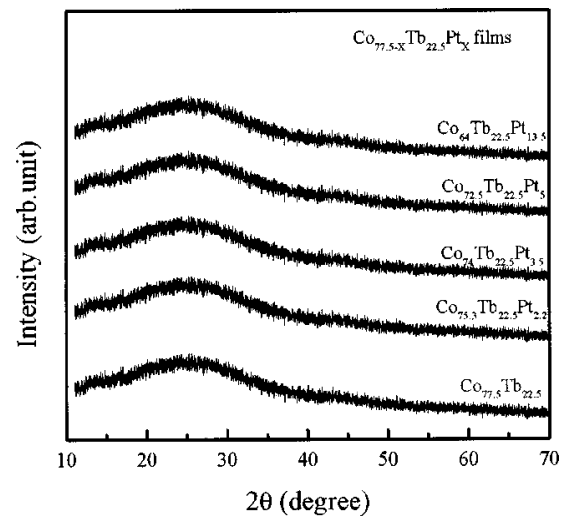

FIG. 1. $\theta-2 \theta$ XRD patterns of various $\mathrm{Co}_{77.5-x} \mathrm{~Tb}_{22.5} \mathrm{Pt}_{x}$ films.

\section{RESULTS AND DISCUSSION}

Figure 1 shows the $\theta-2 \theta$ XRD patterns of various $\mathrm{Co}_{77.5-x} \mathrm{~Tb}_{22.5} \mathrm{Pt}_{x}$ films with different $\mathrm{Pt}$ contents. No film presents a diffraction peak. This implies that all $\mathrm{Co}_{77.5-x} \mathrm{~Tb}_{22.5} \mathrm{Pt}_{x}$ films are amorphous. Figure 2(a) is a typical TEM image of the $\mathrm{Co}_{77.5-x} \mathrm{~Tb}_{22.5} \mathrm{Pt}_{x}$ film, in which $x$ $=13.5$. The film includes no crystal grains. When the specimen is examined in detail, a lemon peel-like microstructure is observed. ${ }^{6}$ Figure 2(b) is the electron diffraction pattern of the film; the broad diffusive diffraction rings indicate that this film has an amorphous structure.

The rare earth-transition metal (RE-TM) magnetic films are easily oxidized. Figure 3 shows the depth profiles of elements in the $\mathrm{Co}_{74.5} \mathrm{~Tb}_{23} \mathrm{Pt}_{2.5}$ film. We can see that $30 \mathrm{~nm}$ $\mathrm{SiN}_{x}$ protective layer is effective to prevent the oxidization of this magnetic film. Some oxygen is present in the two $\mathrm{SiN}_{x}$ protective layers and in the surface of the naturally oxidized $\mathrm{Si}$ substrate but not in the magnetic layer.

Figure 4(a) shows the variations of saturation magnetization, $M_{s}$, and perpendicular remanence, $M_{r}$, with Pt content of the $\mathrm{Co}_{77.5-x} \mathrm{~Tb}_{22.5} \mathrm{Pt}_{x}$ films at room temperature. The $M_{s}$ and $M_{r}$ values of the $\mathrm{Co}_{77.5} \mathrm{~Tb}_{22.5}$ film $(x=0$ at. $\%)$ are about $135 \mathrm{emu} / \mathrm{cm}^{3}$ and $85 \mathrm{emu} / \mathrm{cm}^{3}$, respectively. $M_{s}$ and $M_{r}$ increase rapidly with Pt content $x<2.2$ at. \% . Then, they increase slowly with Pt content when $x>2.2$ at. $\% M_{s}$ exceeds $230 \mathrm{emu} / \mathrm{cm}^{3}$ and $M_{r}$ exceeds $175 \mathrm{emu} / \mathrm{cm}^{3}$ when $x$

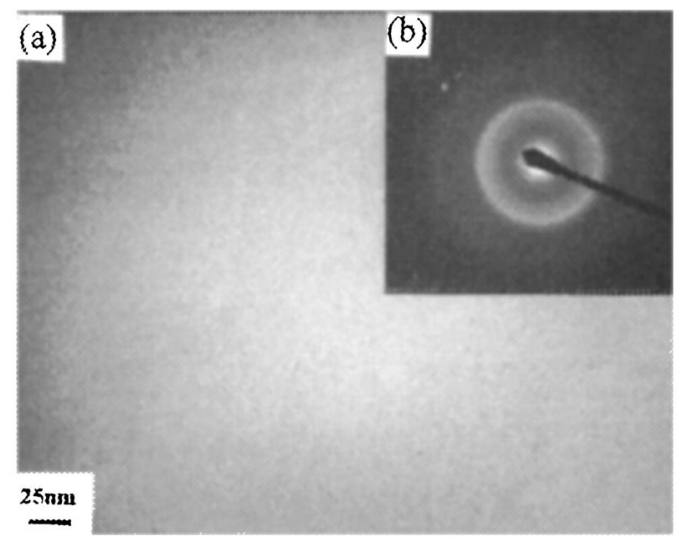

FIG. 2. (a) TEM bright-field image and (b) electron diffraction pattern of the $\mathrm{Co}_{64} \mathrm{~Tb}_{22.5} \mathrm{Pt}_{13.5}$ film.

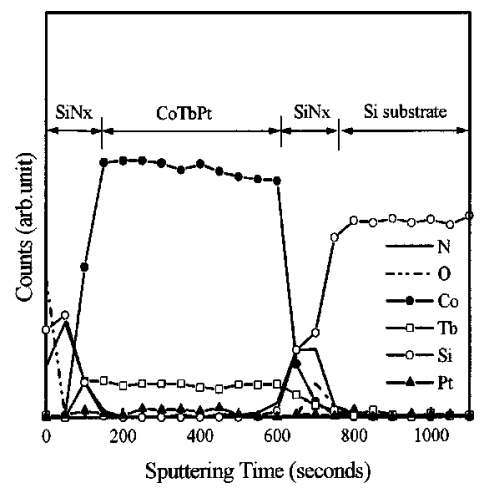

FIG. 3. AES depth profiles of the elements in the $\mathrm{Co}_{75} \mathrm{~Tb}_{22.5} \mathrm{Pt}_{2.5}$ film.

$>2$ at. $\%$. The variation of the squareness, $M_{r} / M_{s}$, with Pt content is very small: The squareness of the film exceeds 0.75 when $x>2$ at. $\%$.

CoTb alloy is sperimagnetic. ${ }^{7}$ The magnetization of the Co subnetwork is antiparallel to that of the Tb subnetwork. The compensation temperature, $T_{\text {comp }}$, of the RE-TM alloy is very sensitive to the composition of the alloy. $\mathrm{Co}_{77.5} \mathrm{~Tb}_{22.5}$ is TM rich because its $T_{\text {comp }}$ is lower than room temperature. ${ }^{8,9}$ The magnetization of the $\mathrm{Co}_{77.5} \mathrm{~Tb}_{22.5}$ alloy is parallel to the direction of Co magnetization. When some Co atoms are substituted for the nonmagnetic Pt atoms, the magnetization of the $\mathrm{Co}_{77.5-x} \mathrm{~Tb}_{22.5} \mathrm{Pt}_{x}$ alloy system declines. So, the $M_{s}$ of the $\mathrm{Co}_{77.5-x} \mathrm{~Tb}_{22.5} \mathrm{Pt}_{x}$ film decreases as the Pt content, $x$, is increased. However, the $M_{s}$ of the $\mathrm{Co}_{77.5-x} \mathrm{~Tb}_{22.5} \mathrm{Pt}_{x}$ film increases with $\mathrm{Pt}$ content, as shown in Fig. 4(a), owing to the effect of the Co-Pt interface on the
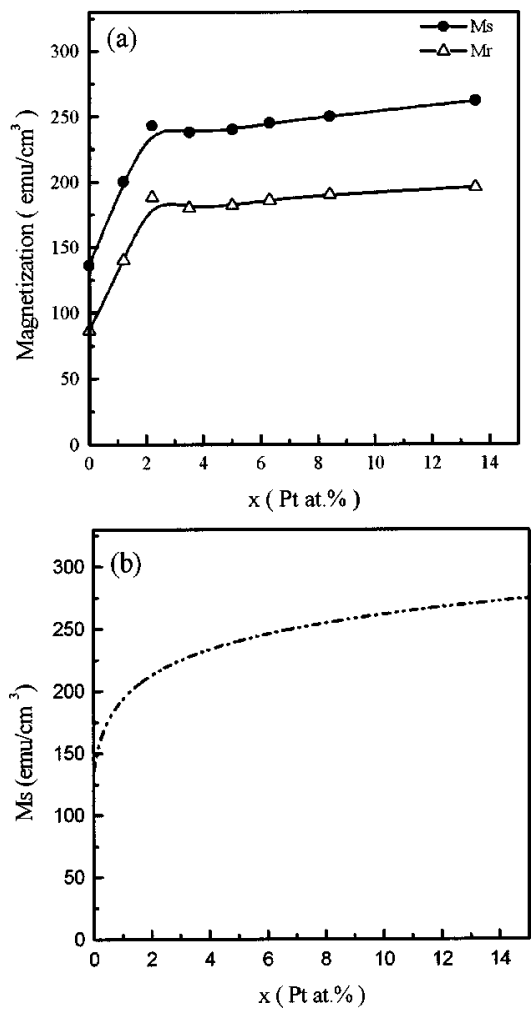

FIG. 4. (a) Variation of $M_{s}$ and $M_{r}$ with Pt content in the $\mathrm{Co}_{77.5-x} \mathrm{~Tb}_{22.5} \mathrm{Pt}_{x}$ film and (b) $M_{s}$ values calculated from Eq. (1). 


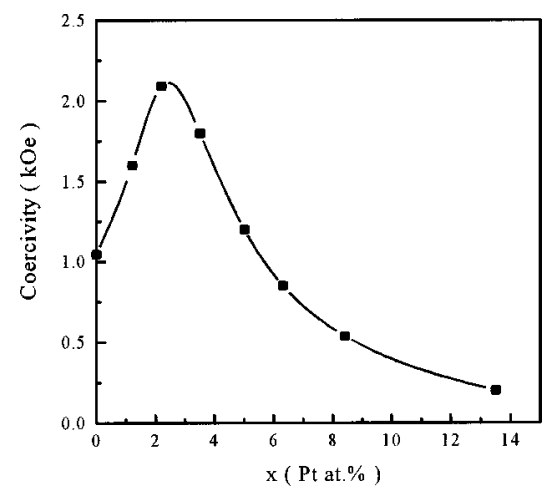

FIG. 5. Variation of $H_{c}$ with $\mathrm{Pt}$ content of the $\mathrm{Co}_{77.5-x} \mathrm{~Tb}_{22.5} \mathrm{Pt}_{x}$ film.

magnetization. ${ }^{10-12}$ The magnetization of Co is increased due to the polarization of the $\mathrm{Pt}$ atoms. The relationship between $M_{s}$ and $x$ of this $\mathrm{Co}_{77.5-x} \mathrm{~Tb}_{22.5} \mathrm{Pt}_{x}$ alloy film can be expressed as

$$
M_{s}=M_{s\left(\mathrm{Co}_{77.5} \mathrm{~Tb}_{22.5}\right)}+\alpha \ln \left(1+x M_{\mathrm{Co}-\mathrm{Pt}} / M_{s(\mathrm{CoTb})}\right),
$$

where $M_{s\left(\mathrm{Co}_{77.5} \mathrm{~Tb}_{22.5}\right)}$ is the saturation magnetization of the $\mathrm{Co}_{77.5} \mathrm{~Tb}_{22.5}$ film $(x=0)$ and $M_{\mathrm{Co}-\mathrm{Pt}}$ is the induced magnetization due to the effect of the Co-Pt interface. $M_{s(\mathrm{CoTb})}$ is the saturation magnetization of $\mathrm{CoTb}$ for the $\mathrm{Co}_{77.5-x} \mathrm{~Tb}_{22.5} \mathrm{Pt}_{x}$ alloy except Pt. $\alpha$ is a constant. Figure $4(\mathrm{~b})$ plots Eq. (1) with the values of the constants chosen to give the best fit to the experimental $M_{s}$ values in Fig. 4(a). The best fit is obtained with $\alpha=30.5 \mathrm{emu} / \mathrm{cm}^{3}$ and $M_{\mathrm{Co}-\mathrm{Pt}} / M_{s(\mathrm{CoTb})}=5.8$. Clearly, $M_{\mathrm{Co}-\mathrm{Pt}}$ can be determined if $M_{s(\mathrm{CoTb})}$ is known.

Figure 5 plots the relationship between perpendicular coercivity $H_{c}$ and the $\mathrm{Pt}$ content of the $\mathrm{Co}_{77.5-x} \mathrm{~Tb}_{22.5} \mathrm{Pt}_{x}$ film at room temperature. The $H_{c}$ value increases from about 1000 Oe to 2100 Oe as Pt content increases from 0 at. \% to 2.2 at. $\%$, and $H_{c}$ decreases rapidly as $x>2.5$ at. $\%$. The increase of $H_{c}$ with Pt content may be owing to that some Pt atoms close together into Pt clusters in the CoTbPt film. These clusters are like defects that cling to the domain wall to reduce the domain-wall energy, ${ }^{13}$ pinning the movement of the domain wall, and increasing the coercivity of the film. ${ }^{14}$ This pinning effect depends on the thickness of the domain wall and the size of the defects. Maximum pinning effect occurs when the size of the defect is about equal to the thickness of the domain wall. ${ }^{13}$ When the Pt content of the film exceeds 2.5 at. \%, the diameter of some Pt clusters may exceed the thickness of the domain wall, reducing the pinning effect. Therefore, the $H_{c}$ value decreases as the Pt content increases as $x>2.5$ at. $\%$.

Figure 6 plots the relationship between the $H_{c}$ value and the temperature of the $\mathrm{Co}_{75.3} \mathrm{~Tb}_{22.5} \mathrm{Pt}_{2.2}$ film, showing that the $H_{c}$ value decreases rapidly from 2100 Oe to about 50 Oe as the temperature increases from $25^{\circ} \mathrm{C}$ to $200{ }^{\circ} \mathrm{C}$. The $M_{s}$

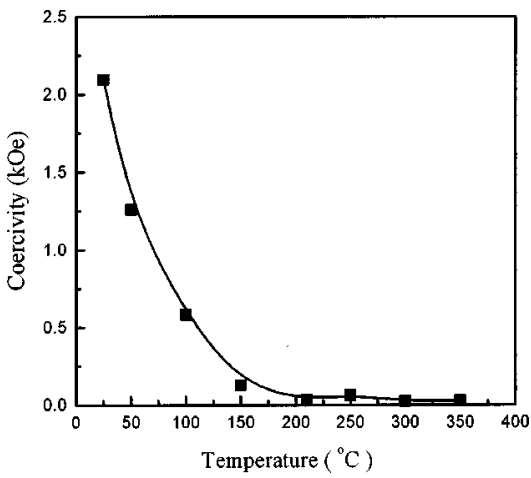

FIG. 6. Relationship between $H_{c}$ and temperature of the $\mathrm{Co}_{75} \mathrm{~Tb}_{22.5} \mathrm{Pt}_{2.5}$ film.

value of the $\mathrm{Co}_{75.3} \mathrm{~Tb}_{22.5} \mathrm{Pt}_{2.2}$ film is about $245 \mathrm{emu} / \mathrm{cm}^{3}$, the squareness is about 0.8 , and the $H_{c}$ is about $2100 \mathrm{Oe}$ at room temperature. The rapid decrease in $H_{c}$ with an increase in temperature is shown in Figs. 5 and 6.

\section{CONCLUSION}

The effects of Pt content on the microstructure and magnetic properties of the $\mathrm{Co}_{77.5-x} \mathrm{~Tb}_{22.5} \mathrm{Pt}_{x}$ films with $x$ $=0-14$ at. $\%$ were investigated. The XRD and TEM analysis indicated that all these films are amorphous. Substituting a small amount of Co for Pt will increases the $M_{s}, M_{r}$, and $H_{c}$ of the $\mathrm{Co}_{77.5} \mathrm{~Tb}_{22.5}$ film.

\section{ACKNOWLEDGMENT}

This work was supported by the National Science Council of Taiwan, R.O.C. through Grant Nos. NSC 90-2216-E 002-036 and MOE 91-EC-17-A-08-S1-0006.

${ }^{1}$ H. Saga, H. Nemoto, H. Sukeda, and M. Takahashi, Jpn. J. Appl. Phys., Part 1 38, 1839 (1999).

${ }^{2}$ J. J. M. Ruigrok, R. Coehoorn, S. R. Cumpson, and H. W. Kesteren, J. Appl. Phys. 87, 5398 (2000).

${ }^{3}$ H. Sukeda, H. Saga, H. Nemoto, Y. Itou, C. Haginoya, and T. Matsumoto, IEEE Trans. Magn. 37, 1234 (2001).

${ }^{4}$ H. Nemoto, H. Saga, H. Sukeda, and M. Takahashi, Jpn. J. Appl. Phys., Part 1 38, 1841 (1999).

${ }^{5}$ C. C. Lin, C. H. Lai, B. M. Chen, and H. P. D. Shieh, IEEE Trans. Magn. 37, 1339 (2001)

${ }^{6}$ P. C. Kuo and C. M. Kuo, J. Appl. Phys. 84, 3317 (1998).

${ }^{7}$ P. Hansen, Handbook of Magnetic Materials, edited by K. H. J. Buschow (Elsevier, New York, 1991), Vol. 6, Chap. 4, p. 310.

${ }^{8}$ M. Mansuripur, The Physical Principles of Magneto-optical Recording (Cambridge University Press, Cambridge, UK, 1995), p. 46.

${ }^{9}$ P. Hansen, S. Klahn, C. Clausen, G. Much, and K. Witter, J. Appl. Phys. 69, 3194 (1991).

${ }^{10}$ J. A. Mydosh and G. J. Nicuwenhuys, Ferromagnetic Materials, edited by E. P. Wohlfarth (North-Holland, Amsterdam, 1980), Vol. 1, p. 71.

${ }^{11}$ P. F. Carcia, J. Appl. Phys. 63, 5066 (1988).

${ }^{12}$ H. Hamouda, H. Lassri, R. Krishnan, and D. Saifaoui, Solid State Commun. 108, 451 (1998).

${ }^{13}$ B. D. Cullity, Introduction to Magnetic Materials (Addison-Wesley, London, 1972), p. 317.

${ }^{14}$ M. Mansuripur, The Physical Principles of Magneto-optical Recording (Cambridge University Press, Cambridge, UK, 1995), p. 46. 